\title{
Glassy cell carcinoma (GCC) of uterine cervix
}

\author{
Rahat Hadi ${ }^{1}$, Milind Kumar ${ }^{2}$, Sushmita Pat ${ }^{2}$, Prasenjit Das ${ }^{3}$, Arvind Vaid ${ }^{4}$, \\ Siddhartha Datta Gupta ${ }^{3}$, B K Mohanti ${ }^{2}$
}

Sri Lanka Journal of Obstetrics and Gynaecology 2011; 33: 65-67

\begin{abstract}
Glassy cell carcinoma (GCC) is a rare clinical entity involving uterine cervix with aggressive course and relatively poor prognosis. The most common presentation is abnormal bleeding or discharge per vaginum of recent origin. Histologically it is diagnosed by characteristic "Glassy" cells. All the three modalities (i.e. surgery, radiotherapy and chemotherapy) have been tried in various clinical settings, showing different clinical outcome. The common consensus regarding diagnosis and optimal treatment modalities is yet to emerge. The case presented here not only represents a rare histopathological diagnosis, but also reemphasizes the importance of Her2 neu immunostains as a bad prognostic marker.
\end{abstract}

Key words: glassy cell carcinoma, radiotherapy, Her2 neu, cervix.

\section{Introduction}

GCC of cervix is considered a rare tumor involving cervix and accounts for around $1 \%$ of all cervical cancers ${ }^{1}$. It is a poorly differentiated subtype of adeno-squamous carcinoma and characterized by glassy cells due to abundant glycogen in the cytoplasm. It has relatively worse prognosis and considered resistant to both radiotherapy and chemotherapy.

\section{Case report}

The patient was a 45-year old menopausal, multiparous woman who presented to our combined

\footnotetext{
${ }^{1}$ Department of Radiation Oncology, Dr Ram Manohar Lohia Institute of Medical Sciences (Dr RMLIMS), Vibhuti Khand, Gomti Nagar, Lucknow-226010, Uttar Pradesh, India.

${ }^{2}$ Department of Radiation Oncology, Dr B R A, Institute Rotary Cancer Hospital (IRCH).

${ }^{3}$ Department of Pathology,

${ }^{4}$ Department of Gynecology and Obstetrics, All India Institute of Medical Sciences (AIIMS), New Delhi110029, India.

Correspondence: Rahat Hadi

E-mail:drrahathadi@yahoo.co.in,drrahathadi@gmail.com
}

gynae-oncology clinic with single complaint of white foul smelling vaginal discharge for last three months. Per speculum examination revealed an exophytic cervical mass approximately $5 \mathrm{~cm}$ in diameter with obliterated fornices. On per-vaginal examination, bilateral parametrium were thickened and reaching up to the lateral pelvic wall. Ultrasonography (USG) abdomen and pelvis revealed an irregular mass of 49.8 $\mathrm{mm} \times 31.7 \mathrm{~mm}$ in cervical area. Contrast Enhanced Computerized Tomography (CECT) abdomen and pelvis showed a heterogeneous, lobulated, enhancing mass in cervix, extending into the lower uterine segment and the upper part of the vagina. The mass was $64 \mathrm{~mm} \times 48 \mathrm{~mm}$ in size towards left side, compressing lower end of the left ureter. It was intending the base and posterior wall of the urinary bladder. Bilateral parametrial streaking was noted. Positron Emission Tomography - Computerized Tomography (PET-CT) revealed uptake in cervix with pelvic and para-aortic nodes.

A tru-cut biopsy was performed from the cervical mass, which showed a malignant tumor comprising of sheets and nests of polygonal to round cells interspersed by a moderate infiltrate of lymphocytes and polymorphs. The clear cell population was involving around $80 \%$ of the total cell population. The cells showed a low nuclear-cytoplasmic ratio (1:3 to $1: 4)$, with centrally situated nucleus with prominent nucleoli and abundant clear to finely granular ground glass cytoplasm (Figures a and b). Many mitotic figures were identified; necrosis was however not identified in this tru-cut biopsy. The cytoplasmic content showed bright eosinophilic positivity with Periodic Acid Schiff stains (PAS), which washed away with diastase treatment, confirming the presence of glycogen (Figure c). The cells showed immunopositivity for vimentin (Figure d), carcinoembryonic antigen and diffuse cytoplasmic and membranous positivity for Her2/neu $(3+)$ (Figure e) and pan-cytokeratin (Figure f). The cells were negative for estrogen (ER) and progesterone (PR) nuclear proteins, HMB45 and S-100 proteins. Based on the histomorphological findings, a diagnosis of GCC was made. The patient was staged according to International Federation of Gynaecology and Obstetrics (FIGO) staging IIIB. The patient was found to be inoperable and her poor general condition precluded the use of chemotherapy in concurrent setting. Hence she was planned for radical radiotherapy alone. In the meantime she deteriorated clinically due to continuous bleeding per vaginum and had developed severe anaemia. She was admitted 
as inpatient and had repeated blood transfusions. In view of her poor general condition, the line of treatment had been changed from radical to palliative. She received palliative radiotherapy with External Beam Radiotherapy (EBRT) 20 Gray (Gy) in 5\# (fractions) in 5 days and was discharged with poor prognosis explained.

\section{Discussion}

GCC was originally described in the cervix by Glücksmann and Cherry in $1956^{2}$. It can occur in various sites, endometrium, fallopian tubes, and colon, with the uterine cervix being the most frequent location. The mean age is around ten years younger than carcinoma cervix patient. Abundant glycogen in the cell cytoplasm, gives them their characteristic glassy appearance (cytoplasm resembles ground glass, hence the name 'glassy cell'). The exact percentage required for diagnosing this lesion is not exactly known, however anywhere from 30 percent to 100 percent of the cells with this 'glassy' appearance in a tumour, makes it a GCC.

Though histomorphology is quite characteristic, the tumor can be missed for large cell non-keratinizing squamous cell carcinoma, lympho-epithelioma like carcinoma and clear cell carcinoma (meso-nephroid carcinoma) of the cervix.

Due to its innocuous presentation, GCC is usually diagnosed at an advanced stage and shows a tendency for pelvic and extra-pelvic metastases even when the primary in cervix is relatively small. Local recurrence usually occurs at the vaginal vault with local spread to parametrium, ovaries and para-aortic lymph nodes ${ }^{3}$. Extra-pelvic metastatic sites includelung, liver, spleen and bone marrow.

Interestingly the tumour cells in GCC can be positive for ER, PR and Her2/neu immunostains as has been reported in literature up to $18.1 \%, 9.1 \%$ and $45.4 \%$ cases respectively ${ }^{4,5}$. In our case though immunohistochemistry for estrogen and progesterone receptors were negative, the cells showed strong diffuse cytoplasmic as well as membranous immunopositivity for Her2/neu (3+ intensity). Her2/ neu over expression have been correlated with aggressive behaviour and a worse clinical outcome. Deshpande et al reported three cases treated with a combination therapy and others with surgery and radiotherapy showed a poor response $e^{6,7}$.

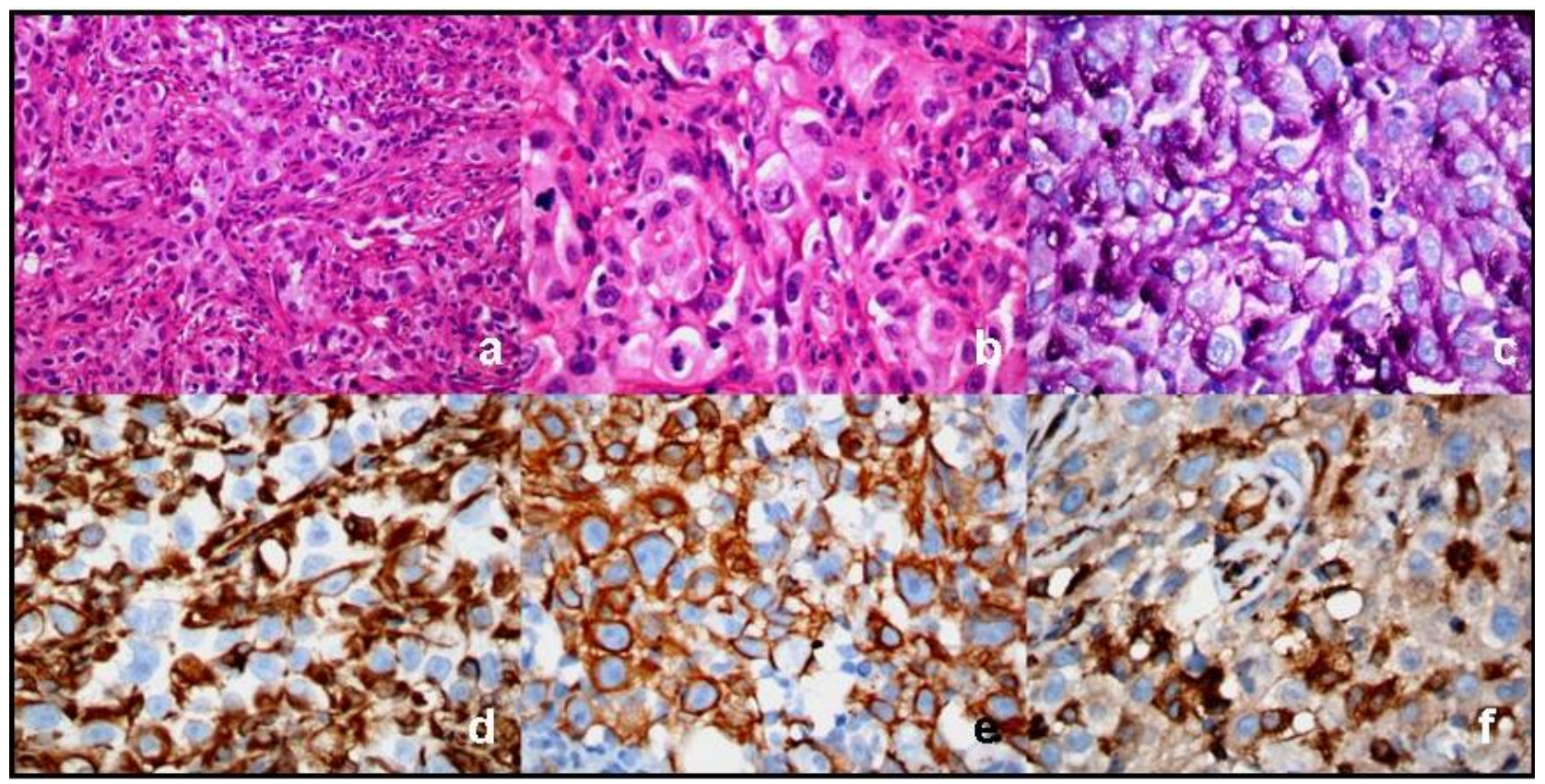

Figures a-f: Photomicrograph demonstrates sheets of polygonal cells with interspersed thin fibrous septae (a, $H \&$ E $\mathbf{4 0}$ ). On higher power the polygonal cells seen to have moderate to abundant amount of thin eosinophilic to clear glassy cytoplasm. The central nucleus shows conspicuous nucleoli. Atypical mitotic figures and scattered polymorphs are also seen in the figure (b, H \& E x 200). On periodic acid Schiff stain the clear cytoplasmic content came out to be strongly PAS positive, indicating the cytoplasmic material as glycogen (c, $H$ \& E x 200). The cells show strong cytoplasmic positivity for vimentin (d, IHC x 200); diffuse strong cytoplasmic as well as membranous positivity for Her2 neu (e, IHC [DAKO, USA] x 200) and also cytoplasmic and membranous positivity for cytokeratin immunostains (f, IHC x 100). 
When radical surgery is combined with radiation therapy and chemotherapy improved overall survival and disease free survival. A retrospective analysis of 22 patients from University of Washington School of Medicine, Seattle, WA, between 1993 and 1999 and at the University of Michigan over a 15 year period showed overall survival of $73 \%(16 / 22)$ and a diseasefree survival of $64 \%(14 / 22)$.

According to the analysis from Rhode Island Hospital, the 28 cases that were treated with bimodal therapy had a disease free survival of $93 \%$ at 2 years and $84 \%$ at 5 years ${ }^{8}$.

Hence multimodality therapy is thought to be preferable in patients with GCC of the cervix even in early stage disease.

\section{References}

1. Lotocki RJ, Krepart GV, Paraskevas M, Vadas G, Heywood M, Fung FK. Glassy cell carcinoma of the cervix: a bimodal treatment strategy. Gynecol Oncol 1992; 44: 2549.

2. Glücksmann A, Cherry MB. Incidence, histology, and response to radiation of mixed carcinoma (adenoacanthomas) of the uterine cervix. Cancer 1956; 9: 971-9.

3. Gray HJ, Garcia R, Tamimi HK, Koh WJ, Goff BA, Greer BE. et al. Glassy cell carcinoma of the cervix revisited. Gynecol Oncol 2002; 85(2): 274-77.

4. Kuroda H, Toyozumi Y, Masuda T, Ougida T, Hanami K, Kyoko K, et al. Glassy cell carcinoma of the cervix: cytologic features and expression of estrogen receptor, progesterone receptor and her2/neu protein. Acta Cytol 2006; 50: 418-22.

5. Chung JH, Koh JS, Lee SS, Cho KJ. Glassy cell carcinoma of the uterine cervix. Cytologic features and expression of estrogen and progesterone receptors. Acta Cytol 2000; 44: 551-6.

6. Piura B, Rabinovich A, Meirovitz M, Yanai-inbar I. Glassy cell carcinoma of the uterine cervix. J Surg Oncol 1999; 72 206-10.

7. Deshpande AH, Kotwal MN, Bobhate SK. Glassy cell carcinoma of the uterine cervix a rare histology: report of three cases with a review of the literature. Indian J Cancer 2004; 41(2): 92-5.

8. Restivo A, Disilvestro P, Moore R, Weitzen S, Kaufman S, Puthawala $Y$, et al. Glassy cell carcinoma of the uterine cervix. ASCO Annual Meeting Proceedings Part I. Journal of Clinical Oncology 2006; 24(18S) (June 20 Supplement): 15050. 